\title{
ENERGY RECOVERY FROM A NON-LINEAR ELECTROMAGNETIC SYSTEM
}

\author{
Krzysztof KĘCIK \\ *Department of Applied Mechanics, Lublin University of Technology, Nadbystrzycka 36, 20-618 Lublin, Poland \\ k.kecik@pollub.pl
}

received 5 April 2017, revised 26 February 2018, accepted 1 March 2018

\begin{abstract}
The paper presents study of a pseudo-magnetic levitation system (pseudo-maglev) dedicated for energy harvesting. The idea rely on motion of a pseudo-levitating magnet in a coil's terminal. The study based on real prototype harvester system, which in the pendulum dynamic vibration absorber is applied. For some parameters, the stability loss caused by the period doubling bifurcation is detected. The coexistence of two stable solutions, one of which is much better for energy harvesting is observed. The influence of the pseudo-maglev parameters on the recovered current and stability of the periodic solutions is presented in detail. The obtained results show, that the best energy recovery occurs for the high pseudo-maglev stiffness and close to the coil resistance. The amplitude's excitation, the load resistances and the coupling coefficient strongly influence on the system's response.
\end{abstract}

Key words: Magnetic Pseudo-Levitation, Energy Harvesting, Vibration, Electromagnetic Coupling, Attractor Coexistence

\section{INTRODUCTION}

Energy harvesting $(\mathrm{EH})$ or energy scavenging $(\mathrm{ES})$ is a promising and evolving field of research capable of supplying power to systems in a broad range of applications. EH can meet in many distinct technologies, including solar panels, wind turbines, kinetic motion harvesters, and thermal generators (Mann and Owens, 2010). The ES devices (harvesters) from vibration have become increasingly widespread because vibrations are everywhere. The devices can be alternative energy sources, especially for microelectromechanical systems (MEMS). The energy recovery from vibration can be achieved by several methods of transform vibration in the electric energy.

Three of the most popular method are:

- Variable Capacitance (VC) Systems,

- Piezoelectric Material (PM) Systems,

- Magnetic Induction (MI) Systems.

The VC systems transduce vibration energy through the electric fields between a parallel plate capacitor with a movable plate. The magnitude of the recovered energy from such systems is generally of the order of microwatts (Jonnalagadda, 2007). The PM systems will produce an electric field and consequently a voltage when deformed under an applied stress. The magnitude of recovered energy from these systems can vary from microwatts to watts (Beeby et al., 2006). Similarly, to the piezoelectric are a magnetostrictive material will produce a magnetic field when deformed. The MI systems also called electromagnetic systems use the motion of a permanent magnet to voltage induction across the terminals of a coil of wire. The magnet's motion creates a magnetic flux leads to the voltage flow in an electrical circuit. The magnitude of recovered energy from these systems can range up to kilowatts, and strongly depending on the size of the magnetic induction systems (Joyce, 2011).

One of the most interesting type of Ml harvesters are the pseudo-maglev systems, which use the pseudo-levitation effect for energy recovery (Mann and Sims 2009; Soares at al., 2016; Sun et al., 2018). The pseudo-levitation harvesters characterize simplicity construction, lack of the dampers and the springs and reliability (Li et al., 2016; Zhou and Wang, 2017). Additionally, can be easy to modification. The pseudo-maglev term means that the magnetic levitation effect occurs with a help mechanical constraint for provides stability. A theorem due to Earnshaw (Earnshaw, 1842) proves that it is not possible to achieve magnetic static levitation using any combination of fixed magnets and electric charges. The static levitation means stable suspension of an object against gravity. From Earnshaw's theorem, at least one stable axis must be present for the system to levitate successfully. However, there are different methods for successful levitation: electromagnets, diamagnetism or superconductors (Qian, 2015; Zhu et al., 2017).

The proposed pseudo-maglev harvester is a crucial component of the laboratory harvester-absorber system, which allows to simultaneously vibration mitigation and energy recovery (Kecik, 2015; Kecik and Mitura, 2016; Kecik et al., 2017a). The detailed study of the maglev harvester helps to proper tune and find the best configuration of the system.

\section{PSEUDO-MAGLEV'S HARVESTER MODEL}

The pseudo-maglev harvester system consists of the permanent cylindrical levitating magnet (moving) suspended in the magnetic field. The scheme and photo of the harvester in Fig.1 are shown. The pseudo-levitation effect coming from the two fixed permanent magnets pairs, which are suitable pole oriented. Each pair of magnets having magnetic poles oriented to repelling NSSN-NS (Fig. 1 (a)). This repel orientation of the magnets is more stable compared to attract magnet's orientation. All magnets are mounted in the cylindrical nonmagnetic tube (made from the nonmagnetic Plexiglas material). The special air holes and gapes are 
made on the tube's surface in order to air cushion reduction. Additionally, the magnet and tube surfaces were sprayed by Teflon to minimalize the friction effect.

a)

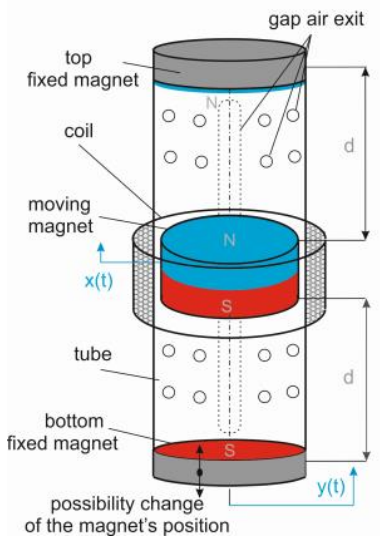

b)

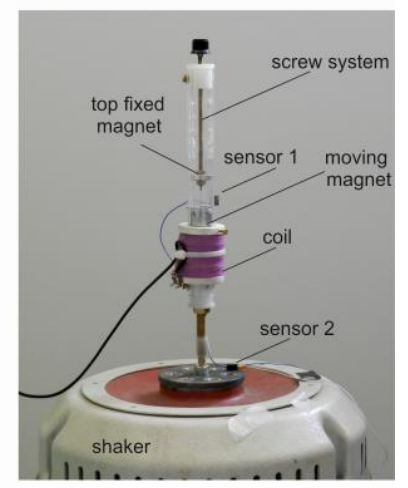

Fig. 1. Pseudo-maglev harvester: a) scheme; b) and prototype (b). This prototype device has been sent to the patent application (no. P.420720)

The small friction and air dissipation is described by the viscous damping coefficients, which were estimated from the free vibration test. The inductor is formed by winding wire around the outer surface of the tube. The distance between the moving and the fixed magnets can be change by the special screw system (see Fig. 1(b)). Modification in the bottom magnet position (parameter $\mathrm{d}$ (Fig.1(a)) causes change in the stiffness suspension (Mann and Sims, 2010).

The pseudo-maglev suspension model has been experimentally identified and proposed in papers (Mann and Sims, 2010; Kecik et al., 2017b), where the obtained results lead to the suspension consists of the non-linear spring and the linear damper. The electromechanical model of the maglev harvester is shown in Fig. 2(a). From a functional principle point of view, the system is similar to a shaker, but the conversion process is reversed.

a)

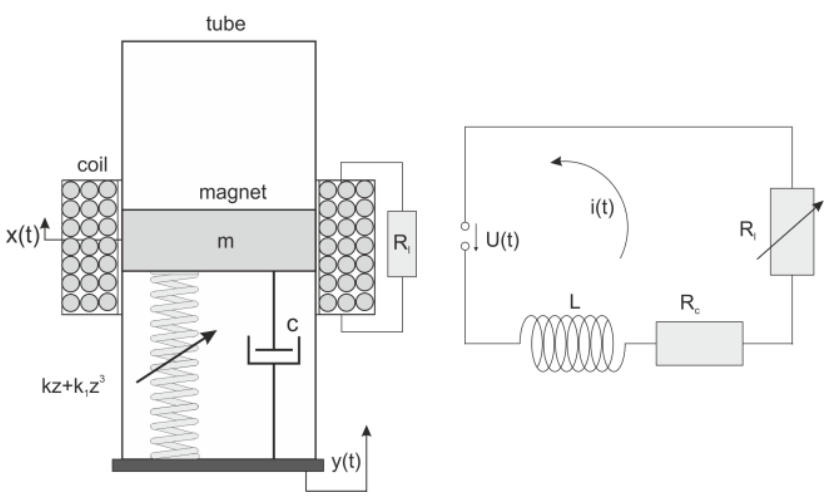

Fig. 2. Schematic diagram of the pseudo-maglev elektromechanical harvester: a) model; b) the electrical circuit

The displacement of the moving magnet from the static position denoted as $x(t)$. The $y(t)$ is the excitation of the base, assumed to be harmonic $y(t)=A \sin (\omega t)$, where parameters $A$ and $\omega$ are the amplitude and the frequency of excitation, respectively. The m means the magnet's mass, $c$ is the viscous damping coefficient, $k$ and $k_{1}$ are the linear and the non-linear stiffness components of the pseudo-maglev suspension. The magnet's oscillation induces current $i(t)$, and voltage $\mathrm{U}(\mathrm{t})$. The electrical circuit of the harvester is presented in Fig. 2(b). It is modelled as the inductor (coil) having the resistance $R_{c}$ and the inductance $\mathrm{L}$. The electrical power generated from the harvester is dissipated across the load resistor $\mathrm{R}_{1}$, which can be adjustable. The total resistance denoted as $R$ means the sum of $\mathrm{R}_{\mathrm{c}}$ and $\mathrm{R}_{\mathrm{l}}$.

The magnetic restoring forces were calibrated from measurements of the restoring force $\mathrm{F}$ and separation distance $\mathrm{d}$ between the bottom and moving magnets with help of the simple static tests. A series of experimental measurements (black points) where the force against a separation distance between two magnets are plotted in Fig. 3.

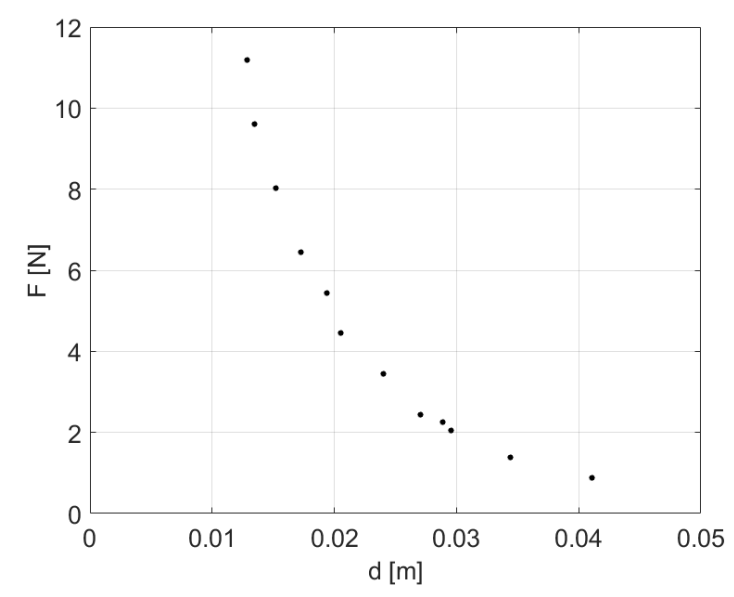

Fig. 3. The restoring force plotted as a function of the separation distance $d$ between the moving and bottom magnet

Next, the experimental results (black points) were transformed into new $x$ coordinates (circle points in Fig. 4). Because the top and bottom magnets are the same, it assumed that both forces are identical. Finally, the curve fitting technique to obtain the mathematical pseudo-maglev suspension model has been used (black line in Fig. 4). On the basis of this result a model of the complete magnetic suspension is developed.

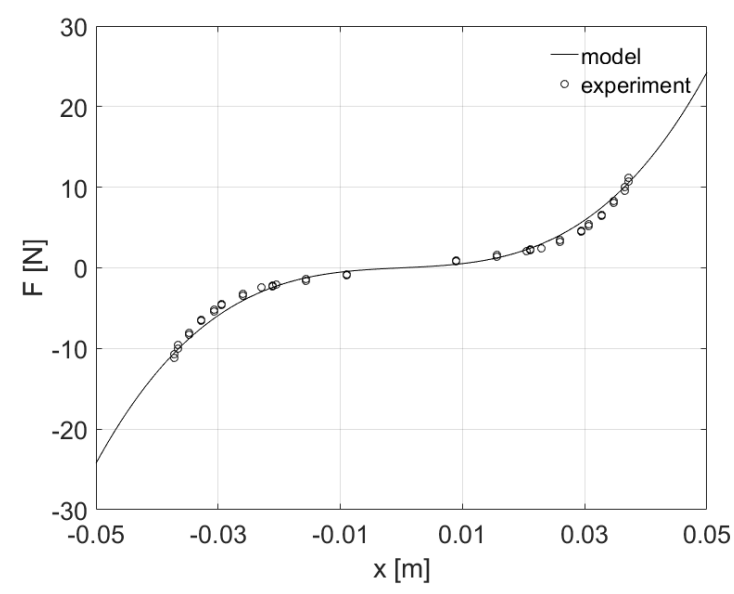

Fig. 4. The force-displacement model magnetic suspension (line) and experimental results (circle points) 
The total restoring force is calculated as a sum of two forces leads to the monostable hard Duffing characteristics

$$
\begin{aligned}
& F(x)=F_{b}(x)-F_{t}(x)=\sum_{n=0}^{3} \beta_{n}(x+d)^{n}- \\
& \sum_{n=0}^{3} \beta_{n}(d-x)^{n}=\left(2 \beta_{1}+4 d \beta_{2}+6 d^{2} \beta_{3}\right) x+2 \beta_{3} x^{3}= \\
& k x+k_{1} x^{3},
\end{aligned}
$$

where $\beta_{0}-\beta_{3}$ are the experimental coefficients estimated from the restoring force (Fig. 3). $\mathrm{F}_{\mathrm{b}}(\mathrm{x})$ and $\mathrm{F}_{\mathrm{t}}(\mathrm{x})$ are the restoring repelling forces describe the relationship between the pairs of magnets (fixed bottom-moving and moving - fixed top). The linear part of the Duffing characteristics is equals $\mathrm{k}=2 \beta_{1}+4 \mathrm{~d} \beta_{2}+$ $6 \mathrm{~d}^{2} \beta_{3}$, and the non-linear component equals $\mathrm{k}_{1}=2 \beta_{3}$. More information about pseudo-magnetic suspension identification technique can be found in (Mann and Sims, 2010; Kecik et al., 2017b), where used very similar methodology.

The mathematical analysis of the pseudo-magnetic suspension characterized by the equation (1) shows two important conclusions:

- the distance $d$ influences on the linear stiffness, and the pseudo-maglev's resonance, only,

- the non-linear part independent on the distance separation of the magnets.

The governing equations of motion for the pseudo-maglev harvester system can be obtained from summation of all forces acting in the vertical direction (simply non-linear oscillator) and use the Kirchhoff's law for the current flow. Additionally, to simplification, the relative magnet's displacement $\mathrm{z}(\mathrm{t})=\mathrm{x}(\mathrm{t})-\mathrm{y}(\mathrm{t})$ is introduced:

$$
\begin{aligned}
& m \frac{d^{2} z}{d t}+c \frac{\mathrm{d} z}{d t}+k z+k_{1} z^{3}+\alpha i+m g=-m \frac{d^{2} y}{d t}, \\
& L \frac{\mathrm{d} i}{d t}+\left(R_{l}+R_{c}\right) i-\alpha \frac{\mathrm{d} z}{d t}=0 .
\end{aligned}
$$

The parameter $\alpha$ is a constant coefficient estimated from the experiment tests (called coupling coefficient). In case of vibration with the small amplitude, the $\alpha$ tends towards zero and the harvester can be treated as linear. Equations (2) and (3) represent two forms of the mathematical model of the non-linear pseudomaglev harvester.

\section{PARAMETER SENSITIVY ANALYSIS}

Before starting the analysis, it is important to clarify goals. To have a general overview how does the electrical parameters change affect on the energy harvesting and dynamics of the pseudo-maglev system, the parametric analysis has been done. These results will allow optimal setting and optimize the harvester system. The numerical calculations have been done in AUTO 07p software (Doedel and Odelman, 2012) using the continuation technique method (pseudo-arclength continuation for following solution families), additionally verified by MATLAB 2015b. The numerical continuation is a numerical method successfully used to response and bifurcation analysis of ordinary differential equation (ODE) systems.

The simulation parameters based on the identification from the laboratory rig presented in Fig. $1(\mathrm{~b})$ and equal: $\mathrm{m}=0.09 \mathrm{~kg}$, $\mathrm{c}=0.054 \mathrm{Ns} / \mathrm{m}, \mathrm{k}_{1}=160000 \mathrm{~N} / \mathrm{m}^{3}, \mathrm{R}_{\mathrm{c}}=1.20 \mathrm{k} \Omega, \mathrm{L}=$
$1.460 \mathrm{H}, \alpha=60 \mathrm{Vs} / \mathrm{m}$. The numerical simulation starts from the fixed point: $\mathrm{z}(\mathrm{t})=0 \mathrm{~m}, \dot{\mathrm{z}}(\mathrm{t})=0 \mathrm{~m} / \mathrm{s}, \mathrm{i}(0)=0 \mathrm{~A}$, and from the periodic solution for coexistence solution detection. The numerical calculations have been performed for the frequency range from $\omega=10 \mathrm{rad} / \mathrm{s}$ to $\omega=100 \mathrm{rad} / \mathrm{s}$, so that the amplitude of excitation force changes along the resonance curves.

\subsection{Amplitude influence analysis}

The exemplary resonance curves of the magnet and the recovered current versus frequency $\omega$ for the various amplitude of excitation A are shown in Fig. 5 and 6, respectively. These diagrams show maximal value of the magnet's displacement $\max (\mathrm{z})$ and the maximal recovered current max(i).

The black line corresponds to the case where the excitation amplitude equals $A=0.005 \mathrm{~m}$, the blue line to $A=0.01 \mathrm{~m}$, and the green line to $A=0.014 \mathrm{~m}$. The bifurcation points are labelled as: SN (saddle-node bifurcation) and PD (period doubling bifurcation). The stable periodic solutions are marked by the solid line, while the dashed-dotted line denotes the unstable solutions.

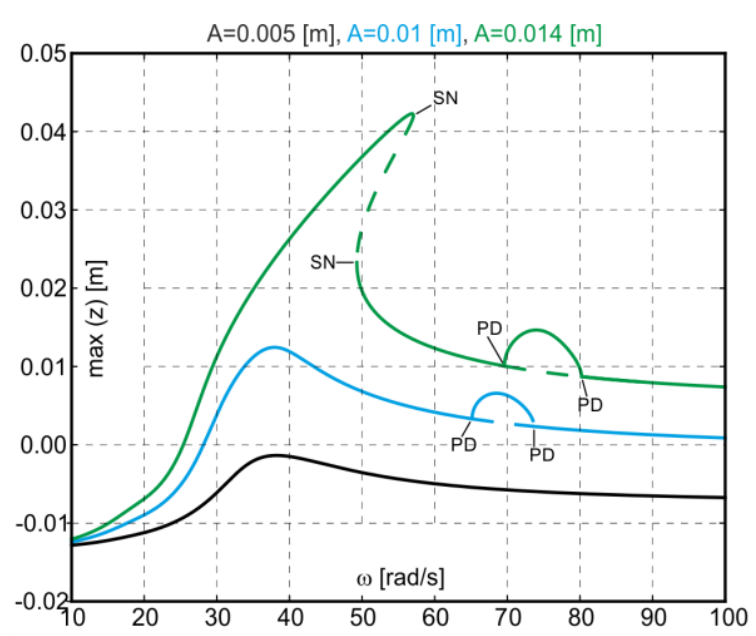

Fig. 5. Resonance curves of the magnet, for $k=38.7 \mathrm{~N} / \mathrm{m}$ and $\mathrm{R}=2.3 \mathrm{k} \Omega$

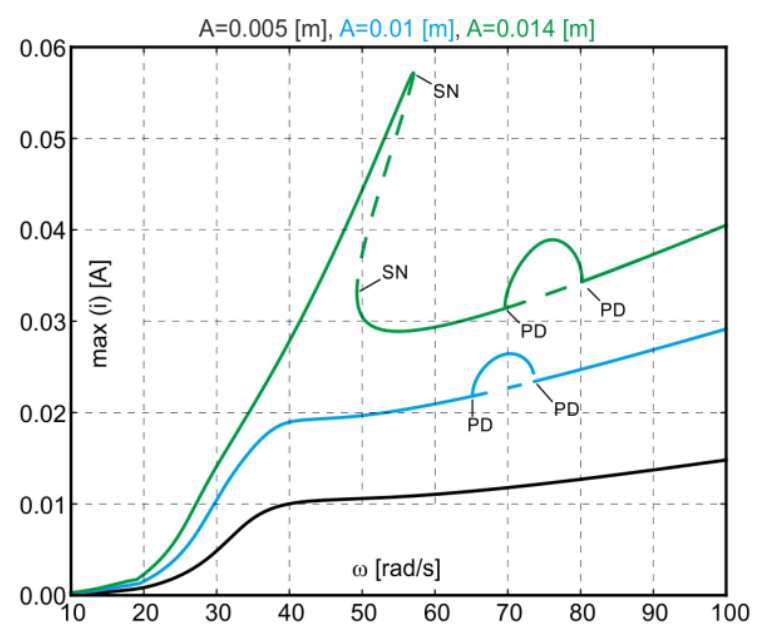

Fig. 6. Recovered current for the various excitation amplitude, for $k=38.7 \mathrm{~N} / \mathrm{m}$ and $\mathrm{R}=2.3 \mathrm{k} \Omega$ 
The resonance peak is observed near the frequency $\omega \approx$ $40 \mathrm{rad} / \mathrm{s}$, but for the higher excitation amplitudes, shape of the resonance curve is distorted (hardening behaviour is observed). The maximal recovered current obtained for the high amplitude of excitation $\mathrm{A}=0.014 \mathrm{~m}$ and equals $\mathrm{i}=0.06 \mathrm{~A}$. Generally, two times greater amplitude causes two times current increase. Moreover, for the higher excitation amplitude, coexistence of two solutions occurs. For frequency range of $\omega \approx 65-75 \mathrm{rad} / \mathrm{s}$ (for $\mathrm{A}=0.01 \mathrm{~m}$ ) and $\omega \approx 70-80 \mathrm{rad} / \mathrm{s}$ (for $\mathrm{A}=0.014 \mathrm{~m}$ ), one stable and one unstable solution are observed.

The new stable solution characterizes change in the vibration period, caused by the PD bifurcation. The period increases from $\mathrm{T}=0.9 \mathrm{~s}$ to $\mathrm{T}=0.18 \mathrm{~s}$. This situation is clearly presented in Fig. 7, for the frequency $\omega=70 \mathrm{rad} / \mathrm{s}$, (black line).

For the large value of $A$, the resonance curve shape is distorted and exhibits two stable periodic solutions (top and bottom branches, Fig. 5 and 6). One of these solutions shows higher energy output.

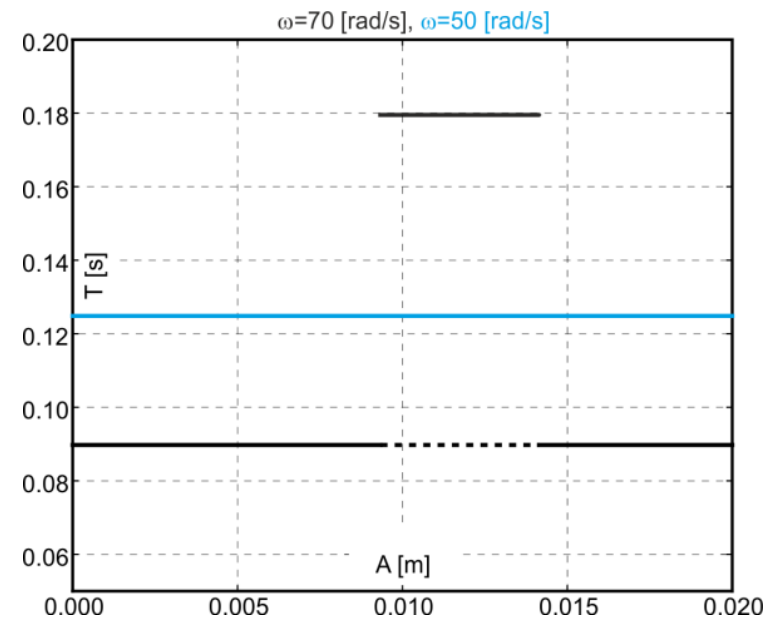

Fig. 7. The change of the magnet vibration period. The black line represents the period doubling bifurcation behaviour

The maximal recovered current equals about $\mathrm{i}=0.06 \mathrm{~A}$ from the top branch, while $\mathrm{i}=0.03 \mathrm{~A}$ from the bottom curve. These solutions depend on the initial conditions of the pseudo-maglev system (initial displacement $\mathrm{z}(0)$ and velocity $\dot{\mathrm{z}}(0)$ ). This effect is called foldover and is characteristic for the non-linear resonances.

The coexistence of two stable solutions are confirmed by the basin of attraction (Fig. 8). Basin of attraction is the set of initial conditions leading to long-time behaviour that approaches that attractor. The red colour with attractor no. 2 denotes the periodic solution corresponding to the bottom branch, while the yellow colour (and its attractor no. 1) corresponds to the top branch of hardening characteristic presented in Fig. 5 . One can easily notice that the BA represents by the yellow colour is dominant. The solution from top branch is much better for the energy harvesting. Therefore, the influence of the electrical parameters is crucial from practical point of view.

Exemplary time series of the pseudo-levitating magnet for $\omega=50 \mathrm{rad} / \mathrm{s}$ is presented in Fig. 9. The blue line means the solution represented by the top branch, while the red line denotes solution from the bottom branch (see, Fig. 5). Note, that both signals have periodic nature.

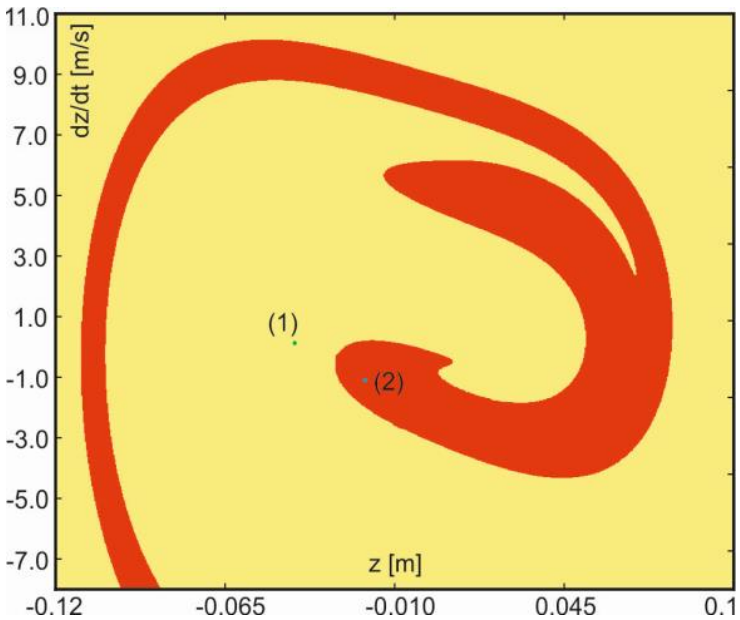

Fig. 8. Basins of attraction for parameters $\omega=50 \mathrm{rad} / \mathrm{s}$ and $A=0.014 \mathrm{~m}$ : (1) attractor of the top branch, (2) attractor of the bottom branch

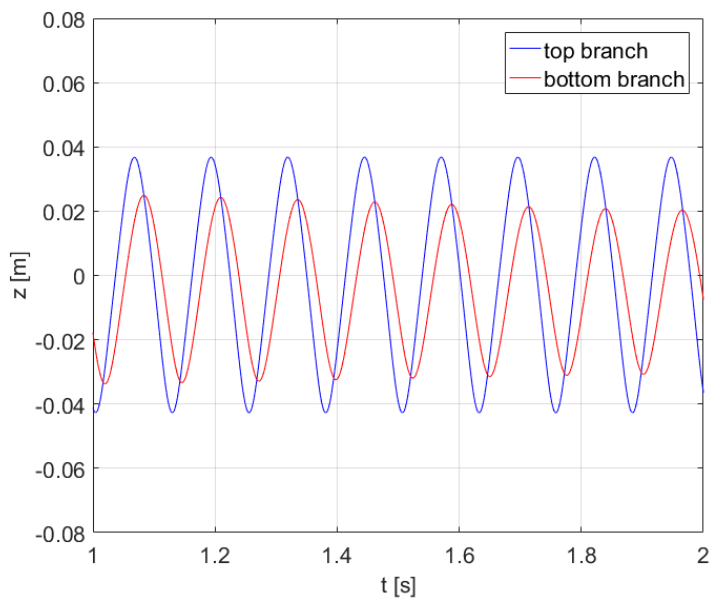

Fig. 9. The magnet vibration vs. time of the top (blue line) and bottom branch (red line), for parameters: $\omega=50 \mathrm{rad} / \mathrm{s}, A=0.014 \mathrm{~m}$

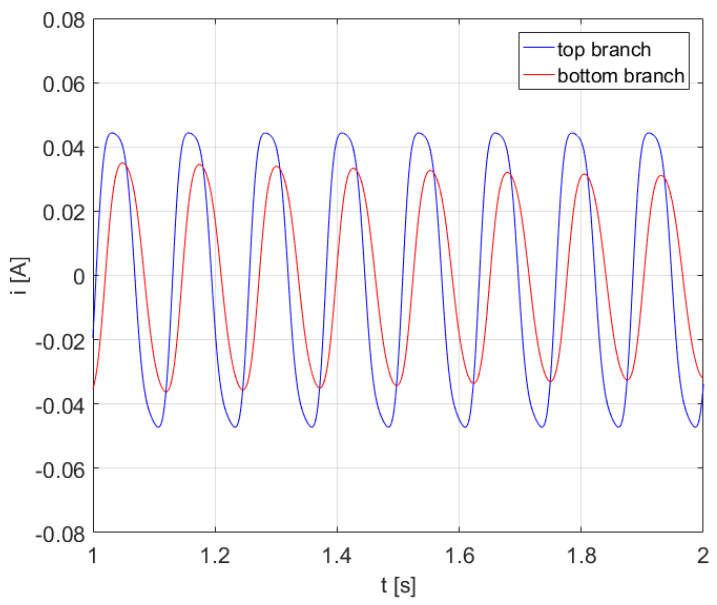

Fig. 10. The recovered current vs. time of the top (blue line) and bottom branch (red line), for parameters: $\omega=50 \mathrm{rad} / \mathrm{s}, A=0.014 \mathrm{~m}$ 


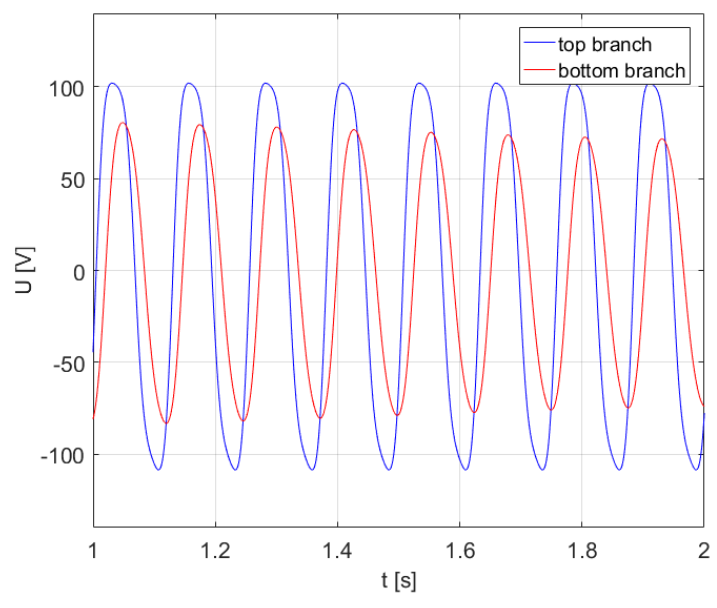

Fig. 11. The recovered voltage vs. time of the top (blue line) and bottom branch (red line), for parameters: $\omega=50 \mathrm{rad} / \mathrm{s}, A=0.014 \mathrm{~m}$

Additionally, the displacement of the vibration centre is observed. This displacement is a result of the magnet's gravitation force, included in equation (2). Of course, the displacement depends on the pseudo-maglev stiffness.

The recovered voltage $U$ and the instantaneous power $P$ extracted from the magnet's vibrations can be computed from simple formulas (Mann and Sims, 2009)

$$
U=i R_{l}, \quad P=i^{2} R_{l} \text {. }
$$

The recovered current and the voltage versus time in Fig. 10 and 11 are presented, respectively. The maximal current value from the top branch equals $\mathrm{i}=0.045 \mathrm{~A}(\mathrm{U} \approx 100 \mathrm{~V})$, while from the bottom is $\mathrm{i}=0.03 \mathrm{~A}(\mathrm{U} \approx 80 \mathrm{~V})$.

Analysing both time series can be concluded that recovered power from the bottom branch equals $\mathrm{P}=2.65 \mathrm{~W}$, while from the top branch is $\mathrm{P}=5.53 \mathrm{~W}$. The recovered current and voltage have periodic course, also. The frequency of the magnet's vibration and the recovered current (and voltage) have the same vibration's period.

\subsection{Pseudo-maglev suspension influence}

The pseudo-magnetic levitation stiffness can be easy modified by the parameter $d$ in the real system. However, the modification also changes the linear resonance of the magnet. The vibration amplitude and amount of the recovered energy strongly depends on parameter $\mathrm{k}$ (Fig. 12). For small stiffness $\mathrm{k}=10 \mathrm{~N} / \mathrm{m}$ the maximal magnet's amplitude equals $\mathrm{z}=0.025 \mathrm{~m}$ and the recovered current equals $i=0.045 \mathrm{~A}$. If the stiffness increases, then the resonance curve exhibit non-linear resonance with the foldover effect. Additionally, the period doubling (PD) region disappears.

For the low frequency level, the parameter $\mathrm{k}$ practically does not influence on the recovered current level. However, increase of $\mathrm{k}$, in higher frequency level the recovered energy is larger (Fig. 13), especially close to the resonance peak $(\omega=40-60 \mathrm{rad} /$ $\mathrm{s})$. For the small pseudo-maglev stiffness $\mathrm{k}=10 \mathrm{~N} / \mathrm{m}$, the higher level of recovered energy occurs in the PD region $(\omega \approx 55-$ $85 \mathrm{rad} / \mathrm{s})$. Interestingly, to this region, that the magnet's oscillation is similar to the vibration in the resonance peak.

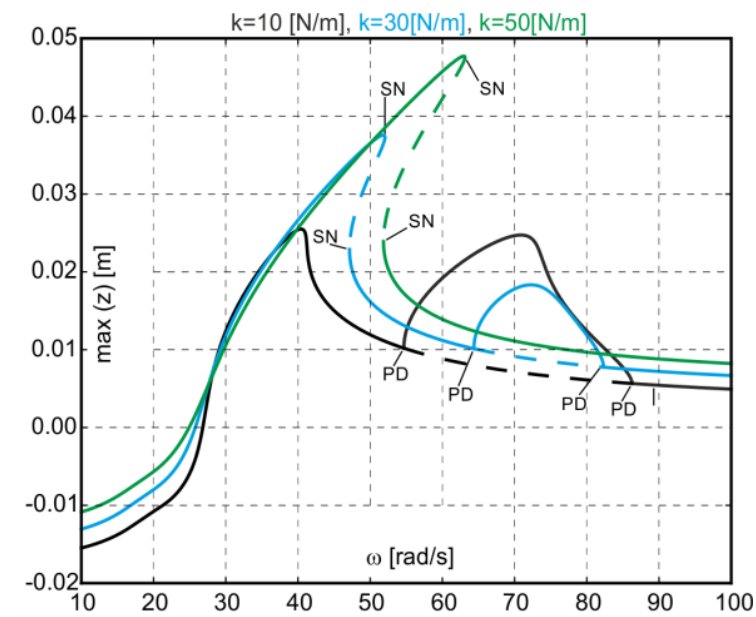

Fig. 12. Resonance curves for various pseudo-maglev stiffness $k$, for parameters: $R=2.3 \mathrm{k} \Omega, A=0.014 \mathrm{~m}$

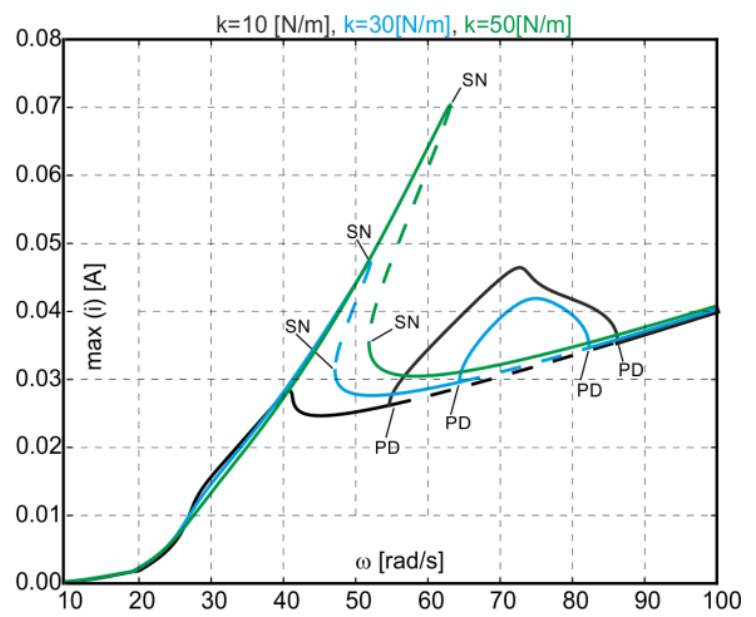

Fig. 13. Recovered current for various pseudo-maglev stiffness $k$, for parameters: $R=2.3 \mathrm{k} \Omega, A=0.014 \mathrm{~m}$

This means that is possible to rise of the recovered energy level without increase of the magnet's oscillation. Note, that the large magnet's oscillations can cause the magnet jump from the coil, but this case was not analysed.

\subsection{Resistance load influence}

The resonance curves for the pseudo-levitating magnet and the recovered current under the load resistance influence $\mathrm{R}$ are shown in Fig. 14 and 15, respectively. The black line corresponds to the case where the total resistance is $R=2.0 \mathrm{k} \Omega$, the blue line to $\mathrm{R}=2.4 \mathrm{k} \Omega$, and the green to $\mathrm{R}=2.8 \mathrm{k} \Omega$. Analysing and comparing the results in both diagrams, it can conclude that resistance R may introduce new solutions. For some parameters, two stable periodic solutions and the loss of the stability caused by PD bifurcation are observed. As expected, the highest recovered energy for the $R=2.4 \mathrm{k} \Omega$ is observed. This means, that the load resistance should be close to the coil resistance $R_{c}=$ $1.2 \mathrm{k} \Omega$. Increase of $\mathrm{R}$ causes that period doubling region is growing. It can see that for small value of $\omega$ the resistance practically does not effect on the magnet's amplitude and the recovered energy. 


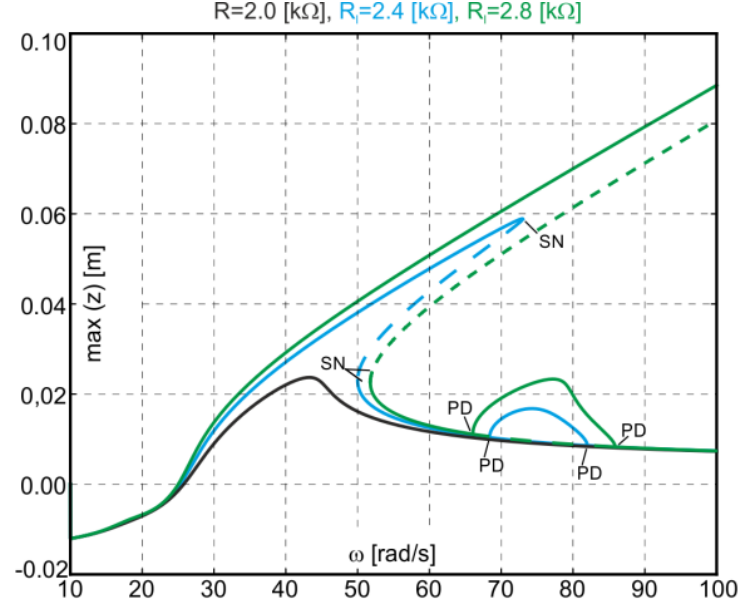

Fig. 14. Resonance curves for the various total resistances, for parameters: $k=38.7 \mathrm{~N} / \mathrm{m}$ and $A=0.014 \mathrm{~m}$

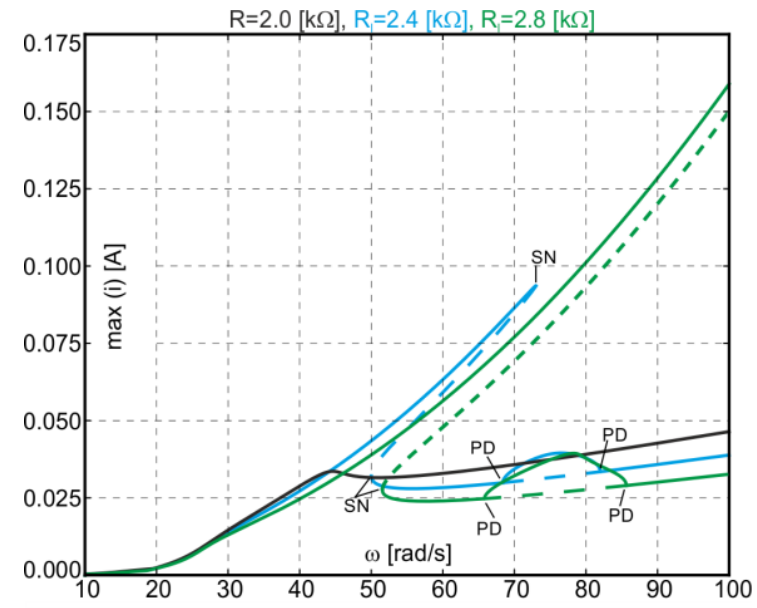

Fig. 15. Recovered current for various total resistances, for parameters: $k=38.7 \mathrm{~N} / \mathrm{m}, A=0.014 \mathrm{~m}$

\subsection{Coil inductance influence}

The coil inductance $\mathrm{L}$ is one of the fundamental parameters describing the coil design. The $\mathrm{L}$ characterizes this behavior of a inductor and is defined in terms of that opposing electromotive force or its generated magnetic flux and the corresponding electric current. The inductance of a circuit depends on the geometry of the current path as well as the magnetic permeability of nearby materials. In our study assumed that the coil inductance is constant, not depends on the magnet's position during current flow. However in most coils terminals have ferromagnetic cores, therefore the inductance will vary with core reluctance, and depends on core length and cross section (and material), positioning and shape (Gomand et al., 2007).

Influence of the coil inductance is shown in Fig. 16 and Fig. 17. The black line corresponds to $\mathrm{L}=0.1 \mathrm{H}$, blue line to $\mathrm{L}=$ $1.46 \mathrm{H}$ and green line to $\mathrm{L}=5.0 \mathrm{H}$. The small values of the inductance (usually typical in real practice) has a negligible effect on the response of the pseudo-maglev system and the energy recovery level.

The influence is visible only for the higher value of the inductance and higher frequency of excitation $(\omega>50 \mathrm{rad} / \mathrm{s})$, where the increase of recovered energy is observable.

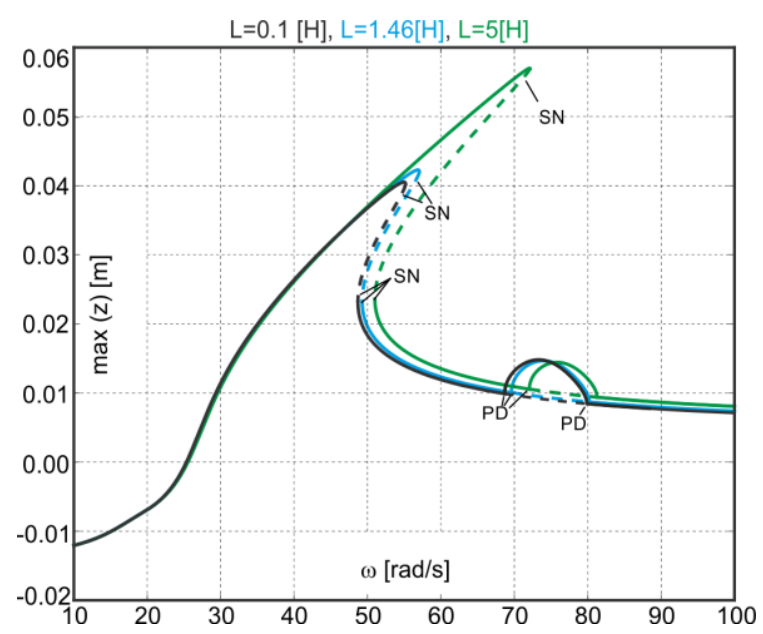

Fig. 16. Resonance curves for various coil inductance, for parameters: $\mathrm{R}=2.3 \mathrm{k} \Omega, \mathrm{k}=38.7 \mathrm{~N} / \mathrm{m}$ and $\mathrm{A}=0.014 \mathrm{~m}$

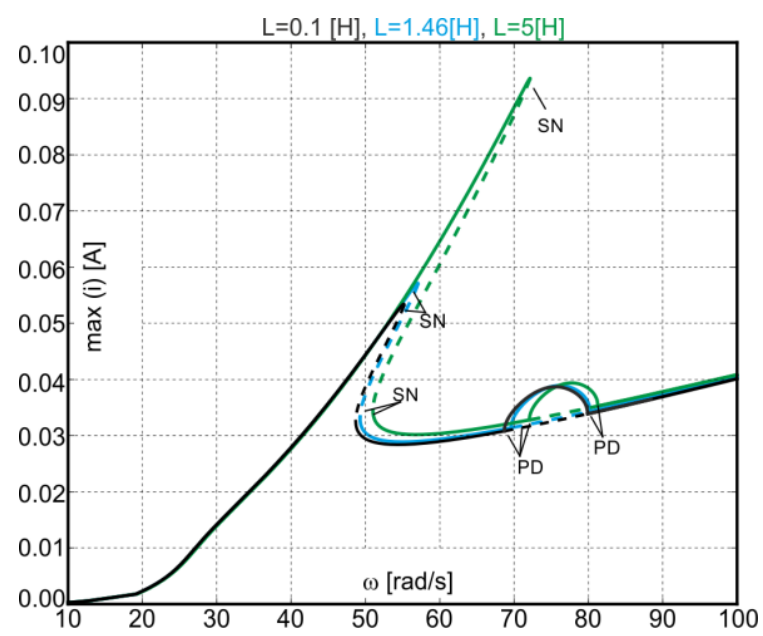

Fig. 17. Recovered current for tvarious coil inductance, for parameters: $\mathrm{R}=2.3 \mathrm{k} \Omega, \mathrm{k}=38.7 \mathrm{~N} / \mathrm{m}, \mathrm{A}=0.014 \mathrm{~m}$

\subsection{Coupling coefficient influence}

The next studied parameter is the coupling coefficient (inductive coefficient). This parameter depends on the magnet position in the coil and construction of the harvester. However, the paper (Kecik et al., 2017b) shows, that constant coupling coefficient value can be accepted, if it is properly chosen.

The blue line shows the resonance curves obtained for $\alpha=60 \mathrm{Vs} / \mathrm{m}$ and it is value chosen from the experiment based on the static tests and calculated from Faraday's law. If the coefficient increases, then the magnet's amplitude and the recovered energy are significantly reduced (green lines in Fig. 18 and 19). Otherwise, if the coefficient decreases, then magnet's amplitude and the recovered current have higher level and the PD region expanded. This means that $\alpha$ plays role of the electrical damping. Interestingly, that for the low frequency, three stable solution are observed (nearly $\omega \approx 85 \mathrm{rad} / \mathrm{s}$ ).

In many papers (Williams and Yates, 1996; Beeby et al., 2006; Olaru et al., 2014), the inductance $L$ is neglected, then the coupling coefficient $\alpha$ is additional damping comes from the electrical circuit. Often researchers have treated the magnetic flux density as uniform over the coil volume and constant over the 
magnet's entire range of motion and this factor depends on the magnitude of the magnetic flux and the length of wire composing the coil (Mitcheson et al., 2004; Mitcheson, 2005; Bedekar, 2009).

The new model of the coupling coefficient and compared it with the classical approach is presented in paper (Kecik et al., 2017b).

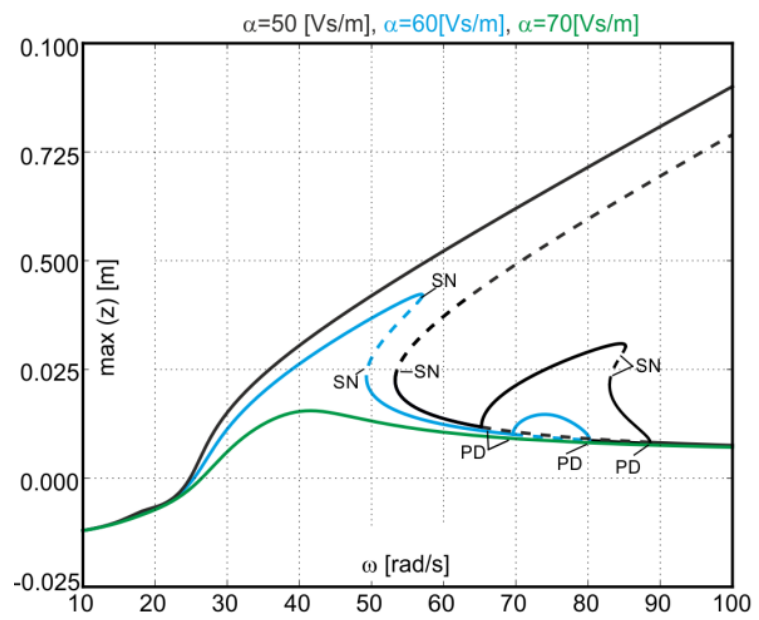

Fig. 18. Resonance curves for various coupling coefficient, for parameters: $R=2.3 \mathrm{k} \Omega, k=38.7 \mathrm{~N} / \mathrm{m}, A=0.014 \mathrm{~m}$

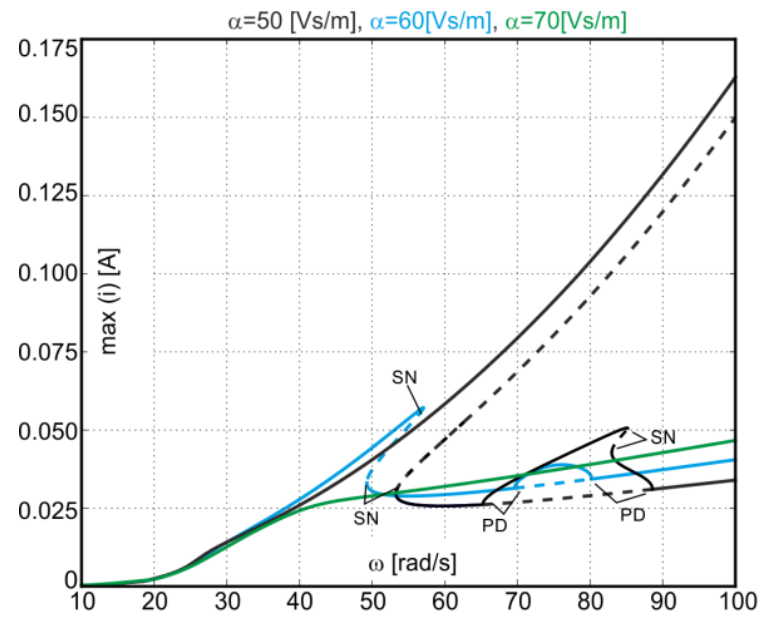

Fig. 19. Recovered current for various coupling coefficient, for parameters: $R=2.3 \mathrm{k} \Omega, k=38.7 \mathrm{~N} / \mathrm{m}, A=0.014 \mathrm{~m}$

\section{CONCLUSIONS AND FINAL REMARKS}

The paper presents numerical study of the prototype electromagnetic pseudo-levitation harvester. The influence of the main system's parameters on the recovered current and dynamics of the moving magnet have been presented.

The obtained results show interesting behaviour especially for the higher level of excitation, like the stability loss, the period doubling bifurcation and the attractor coexistence. The maximal recovered current equals about $\mathrm{i}=0.17 \mathrm{~A}$, what corresponds to the power about $\mathrm{P}=66 \mathrm{~W}$.

The load resistance and the coupling coefficient similarly influence on the system's response, causes bending the resonance curves (hardening behaviour) and the foldover effect is observed. The coupling coefficient strongly influences on the recovered current level, therefore should be proper estimated.

For the higher frequency, the coexistence or two or more periodic solutions are possible. The coexistence of solution is promising from the energy harvesting point of view, because one solution (top branch) recover even six times more energy.

The electrical parameters of the pseudo-maglev harvester system can be applied to energy harvesting of small devices and the magnet's vibration control.

The next step will be experimental verification of the obtained results and elaboration the control methods to jump from the lower to high energy input solution. Additionally, the optimization problem will be studied.

\section{REFERENCES}

1. Bedekar V., Oliver J., Priya S. (2009), Pen harvester for powering a pulse rate sensor, Journal of Physics D: Applied Physics, 42(10), 105105.

2. Beeby S., Tudor M., White N. (2006), Energy harvesting vibration sources for microsystems applications, Measurement Science and Technology, 17(12), 175-195.

3. Beeby S.P., Torah R.N. Tudor M.J. (2008), Kinetic energy harvesting. ACT Workshop on Innovative Concepts. ESA-ESTEC 17, 1-10.

4. Doedel E., Oldeman B. (2012), Auto-07p: Continuation and bifurcation software for ordinary differential equations, Concordia University, Montreal, 1-266.

5. Earnshaw S. (1842). On the nature of the molecular forces which regulate the constitution of the luminiferous ether, Transactions of the Cambridge Philosophical Society, 7, 97-112.

6. Gomand J., Remy G., Tounzi A., Barre P.J., Hautier J.P. (2007), Impact of permanent magnet field on inductance variation of a PMLSM, European Conference on Power Electronics and Applications, 1-10.

7. Jonnalagadda A.S. (2007) Magnetic induction systems to harvest energy from mechanical vibrations, $\mathrm{PhD}$ thesis, Massachusetts Institute Engineering.

8. Joyce S. (2011) Development of an electromagnetic energy harvester for monitoring wind turbine blades, PhD thesis, Virginia Polytechnic.

9. Kecik K. (2015) Dynamics and control of an active pendulum system, International Journal of Non-linear Mechanics, 70, 63-72.

10. Kecik K., Brzeski P., Perlikowski P. (2017a) Non-linear dynamics and optimization of a harvester absorber system, International Journal of Structural Stability and Dynamics, 17(9), 1-15.

11. Kecik K., Mitura A. (2016), Nonlinear dynamics of a vibration harvest-absorber system. Experimental Study, Springer Proceedings in Mathematics \& Statistics, Dynamical Systems: Modelling, 181, 197-208.

12. Kecik K., Mitura A., Lenci S., Warminski J. (2017b), Energy harvesting from a magnetic levitation system, International Journal of Non-linear Mechanics, 94, 200-206.

13. Li Y.J., Dai Q., Zhang Y., Wang H., Chen Z., Sun R.X., Zheng J., Deng C.Y., Deng Z.G. (2016), Design and analysis of an electromagnetic turnout for the superconducting Maglev system Physica C: Superconductivity and its Applications, 528, 84-89.

14. Mann B., Sims N. (2010), On the performance and resonant frequency of electromagnetic induction energy harvesters, Journal of Sound and Vibration, 329(1-2), 1348-1361.

15. Mann B.P. Sims N.D. (2009), Energy harvesting from the nonlinear oscillations of magnetic levitation, Journal of Sound and Vibration, 319(1-2), 515-530.

16. Mann B.P., Owens B.A. (2010), Investigations of a nonlinear energy harvester with a bistable potential well, Journal of Sound and Vibration 329, 1215-1226.

17. Mitcheson P.D. (2005), Analysis and optimisation of energyharvesting micro-generator systems, University of London. 
23. Williams C., Yates R. (1996), Analysis of a micro-electric generator for microsystems, Sensors and Actuators A: Physical, 52 (1-3) 8-11.

24. Zhou D, Yu P., Wang L, Li J. (2017), An adaptive vibration control method to suppress the vibration of the maglev train caused by track irregularities, Journal of Sound and Vibration, 408(10), 331-350.

25. Zhu H., Khiang Pang Ch., Joo Teo T. (2017), Analysis and control of a 6 DOF maglev positioning system with characteristics of endeffects and eddy current damping, Mechatronics, 47, 183-194.

Acknowledgments: This work was financially supported under the project of National Science Centre according to decision no. DEC2013/11/D/ST8/03311.

Sun R., Zheng J., Zheng B., Qian N., Li J., Deng Z. (2018), New magnetic rails with double-layer Halbach structure by employing $\mathrm{NdFeB}$ and ferrite magnets for HTS maglev, Journal of Magnetism and Magnetic Materials, 445, 44-48. 